\title{
QUALITY OF LIFE IN BREAST CANCER SURVIVORS: AN ASSESSMIENT OF INTERNATIONAL BREAST CANCER DRAGON BOAT RACERS
}

\author{
L. Koehler, S. Rosenberg, J. Cater, K. Mikolajczyk, \\ A. Moran, C. Metz, S. Monson
}

Division of Physical Therapy (LK,KM,AM,CM,SM) and Rehabilitation Science (LK), Rehabilitation Medicine Department, University of Minnesota Medical School, Minneapolis; Masonic Cancer Center (LK), University of Minnesota, Minneapolis; M Health Fairview, Vascular, Vein, and Wound, Maplewood, Minnesota (SR), Department of Physical Medicine and Rehabilitation, University of Minnesota Medical School, Minneapolis (SR); Abbott Northwestern Hospital (JC), Minneapolis; St. Paul Dragon Divas Dragon Boat Team St. Paul, Minnesota (JC, SR), USA

\section{ABSTRACT}

Resistance exercise is deemed safe for women recovering from conventional breast cancer therapies but few clinicians are aware that dragon boat racing, as a form of resistive exercise, is available to the breast cancer community. The objectives of this study were to 1) increase clinician awareness of dragon boat racing $(D B R)$ in breast cancer survivors as a community-based physical activity, and 2) evaluate quality of life (QOL) in breast cancer survivors with or without lymphedema who participate in DBR. This prospective, observational study surveyed 1,069 international breast cancer dragon boat racers from eight countries to compare function, activity, and participation in women with and without selfreported lymphedema using the Lymph-ICF questionnaire. Seventy-one percent of women ( $n=758$ ) completed the questionnaires. Results revealed significantly higher Lymph-ICF scores in the lymphedema participants, signifying reduced QOL, when compared to the nonlymphedema participants ( $p<0.05$ ), except for "go on vacation" for which no statistical difference was reported ( $p=0.20)$. International breast cancer survivors with lymphedema participating in DBR at an international competition had reduced function, limited activity, and restricted participation compared to participants without lymphedema.

Clinicians should consider utilizing DBR as a community-based activity to support exercise and physical activity after a breast cancer diagnosis.

Keywords: breast cancer, dragon boat racing, DBR, lymphedema, exercise, quality of life, Lymph-ICF

Lymphedema is a post-surgical complication of breast cancer subsequent to lymph node removal. Lymphedema presents as chronic swelling in the arm and/or trunk on the surgical side. This may lead to significant physical, psychological and social problems which impact quality of life (QOL) (1). Previously, health care providers recommended breast cancer survivors avoid upper extremity repetitive resistance exercise, including basic activities of daily life like gardening and cooking, out of concern strenuous exercise would induce inflammation and exacerbate lymphedema (2). In 1998, McKenzie published the first seminal study to debunk this myth 
demonstrating that breast cancer survivors could safely participate in strenuous exercise without causing or worsening lymphedema symptoms (3). This study generated an increase in popularity for breast cancer survivors to participate in dragon boat racing (DBR) and perpetuated a critical turning point for clinicians and researchers to allow breast cancer survivors to participate in resistance exercise. Health care providers have changed practice guidelines and no longer recommend avoidance of exercise in their patients with breast cancer. Researchers have since launched numerous studies analyzing the effects of exercise in breast cancer survivors (4-10).

In the past 20 years, DBR has become a rapidly growing community-based activity gaining international popularity among breast cancer survivors. The International Dragon Boat Federation (IDBF), the governing body for worldwide dragon boat races, recognizes a separate division specifically made for survivors of breast cancer to provide competitions specific to breast cancer survivors. The International Breast Cancer Paddlers' Commission (IBCPC) Participatory Dragon Boat Festival is the largest of these competitions, which provided the unique opportunity to gather data from a large cohort of international breast cancer survivors participating in DBR.

The purpose of this community based participatory research project was to 1 ) increase clinician awareness of DBR in breast cancer survivors as community-based resource for exercise and physical activity, and 2) compare impairments in function, activity limitations, and participation restrictions in international breast cancer survivors with and without lymphedema who participate in competitive DBR. A priori, we hypothesized dragon boat racers without lymphedema will have significantly better Lymph-ICF scores compared to those with lymphedema.

\section{MATERIALS AND METHODS}

The project was a community based participatory research study consisting of a partnership between the University of
Minnesota/Masonic Cancer Center and a local breast cancer dragon boat team from St. Paul, MN. In partnership, the study was designed to be a prospective, observational design comparing the function, activity, and participation of breast cancer dragon boat racers with self-reported lymphedema to those without lymphedema who participated in the IBCPC festival in Sarasota, FL. This study was reviewed by the University of Minnesota Internal Review Board Human Subjects Committee and determined the study was exempt from full review under federal guidelines 45 CFR Part 46.101(b), category 2. Therefore, consent was waived, and women chose to participate in the study if they willingly completed and returned the surveys to the distributing team. Inclusion criteria included women with a history of breast cancer that dragon boat race and attended the IBCPC festival in Sarasota, FL. Women unable to read English were excluded from the study. The St. Paul DBR team selected the questionnaire for the IBCPC Festival during a routine meeting. The primary investigator $(\mathrm{PI})$ provided a choice of established QOL and function questionnaires to the DBR team. The team members then chose the Lymph-ICF based off of content, ease of use, and time to administer the questionnaire. The PI requested and was granted permission from the author of the Lymph-ICF to use the questionnaire for distribution at the festival.

\section{Lymphedema Functioning, Disability and Health Questionnaire (Lymph-ICF)}

The Lymph-ICF is a valid and reliable tool for assessing impairments in function, activity limitations and participation restrictions in patients with arm lymphedema (11). The Lymph-ICF is a 29 item visual analog scale (VAS). Participants mark on the scale from 0 - 100, their average score experienced during the previous two weeks in five different domains. These domains include: (1) physical function, (2) mental function, (3) household activities, (4) mobility activities and (5) life and social activities. The total score on the Lymph-ICF is calculated by 
summating the scores on all the questions and dividing by the total number of answered questions. Scores can also be determined for each of the 5 domains by summating the scores from the questions in the domain divided by the total number of answered questions in the associated domain.

\section{Dragon Boat Racing in Breast Cancer Survivors}

DBR is a competitive team activity requiring intense repetitive exercise. It is a rapidly growing sport with international popularity that is increasing among breast cancer survivors following McKenzie's seminal study (3). McKenzie put a group of breast cancer survivors from Canada through intense repetitive and resistive training to compete in dragon boat racing (DBR). No new cases of lymphedema occurred and none of the existing cases became worse in this group of dragon boat racers demonstrating breast cancer survivors could safely participate in strenuous exercise such as DBR (3).

The IBCPC Participatory Dragon Boat Festival was held in Sarasota, Florida in 2014. The IBCPC hosted 2500 registrants from 102 teams representing at least 9 countries. Most teams consisted of at least 20 paddlers, 1 drummer, and 1 steersperson. The paddling technique integrates repetitive full body, synchronous movements requiring consistent strength and cardiovascular training of all team members. Races usually vary from 250 to 2000 meters with $\mathbf{5 0 0}$ meters being a standard distance for more competitive festivals. A 500 meter race typically takes 2-3 minutes depending on the crew. Races of 200-500 meters are most common at festivals and are considered sprints, ranging from about 1 - 3 minutes to complete. Breast cancer survivor teams welcome racers of all stages of disease, including advanced disease, to participate.

\section{Self-Report of Lymphedema}

Lymphedema was identified by selfreport based on the written question, "Have you ever been diagnosed with lymphedema or experienced swelling in the arm, hand, shoulder, or upper body since diagnosed with cancer?" Individuals were separated into two groups, (1) lymphedema and (2) non lymphedema based on their self-reported response.

\section{Questionnaire Acquisition}

Lymph-ICF scores and individual characteristics, such as self-report of lymphedema, cancer history, and country of residence, were gathered utilizing a paper format questionnaire. Members of the St. Paul DBR team (Dragon Divas of St. Paul) who took part in the IBCPC Participatory Dragon Boat Festival held in Sarasota, FL distributed and retrieved the paper questionnaires at the festival. Prior to delivering the questionnaires to the University of Minnesota, the DBR team discarded blank questionnaires without any markings. Members of the St. Paul team were excluded from participating in the study to avoid potential bias.

Once the questionnaires were delivered to the research lab, the PI managed and stored the data and representatives from the St. Paul DBR team and DPT students from the PI's lab assisted with measuring and data entry. The Lymph-ICF VAS scales were double measured and double entered, and the participant history responses were double entered. Responses that were difficult to read or interpret were flagged and reviewed by the PI, at minimum, and most often also reviewed by a second person from the research team for final interpretation. Data entry for the Lymph-ICF scores were entered separately from the patient history responses then linked together after data entry was complete.

\section{Data Reduction and Statistical Analysis}

The research team strived to maximize representation of the DBR breast cancer survivors by minimizing data reduction since one of the primary goals of the study was to increase awareness of DBR in breast cancer survivors. Therefore, the number of individuals used for each statistical analysis was different dependent on the available data. 
Participant characteristics: The criteria to analyze participant characteristics required a response to the self-report lymphedema question and a minimum of 1 item answered on the Lymph-ICF questionnaire. This allowed the research team to maximize the use of the full data set for analyses which was an important condition for the dragon boat race breast cancer community.

Group difference (lymphedema vs. no lymphedema) in Lymph-ICF scores (individual questions, each domain, and total score): Lymph-ICF questions were analyzed individually for all questions answered (even if only one question was answered) if the participant also responded to the self-report lymphedema question. Since the number of missing items allowed on the Lymph ICF domains and total scores is unknown, missing data was not allowed for the Lymph-ICF total score and each of the domain analyses. To be included in the Lymph-ICF total score group analysis, all 29 Lymph-ICF questions had to be answered. Group analysis for each domain required all questions to be answered within that domain (physical function=7 questions, mental function $=4$ questions, household activities $=4$ questions, mobility activities $=8$ questions, life and social activities $=6$ questions).

Statistical analysis was performed by the PI using NCSS 11 Statistical Software (NCSS 2016, LLC. Kaysville, Utah, USA, ncss.com/software/ncss.) A two sample student t-test compared group differences in LymphICF scores for each question, domain, and total scores and years since surgery in women who self-reported lymphedema to women who selfreported not having lymphedema. If data were not normally distributed or homogeneity of variance was violated, the Mann-Whitney $U$ non-parametric test was used.

Analysis of variance (mixed model) assessed between group (lymphedema/nonlymphedema) and differences in Lymph ICFscores and their interactions. The Mauchly test statistic checked for homogeneity of variance. If violated, the $p$-value was corrected using the Geisser-Greenhouse correction. United States, Australia, and Canada were the only countries with adequate data to run this analysis. Descriptive statistics were used to describe the other countries that did not have adequate data (i.e. 3 or less in a group).

Sample size: Although this was a convenience sample size, a priori an estimated sample size of 98 (24 lymphedema:74 no lymphedema) was calculated to provide $95 \%$ power to determine a 12 point difference in Lymph-ICF scores with a standard deviation of 14 using a p-level of 0.05 .

\section{RESULTS}

Approximately 2,000 written questionnaires were brought to the IBCPC Festival in Sarasota, FL for distribution. Before returning, members from the St. Paul team discarded questionnaires that were blank to minimize the burden of traveling with blank questionnaires. A total of 1,069 questionnaires were returned to the University of Minnesota. Seventy-one percent of the returned questionnaires were sufficiently completed; therefore, final analysis included $\mathbf{7 5 8}$ questionnaires.

Returned surveys represented eight different countries with the majority of surveys representing the United States (39.37\%), Australia (38.31\%), and Canada (11.10\%). Other countries included United Kingdom (3.30\%), Italy (1.72\%), Ireland $(0.92 \%)$, South Africa $(0.79 \%)$, and New Zealand $(0.79 \%)$. The name of the country was not reported in $3.7 \%$ of the questionnaires returned. Participant characteristics are presented in Table 1. Lymphedema group differences were found in cancer recurrence, chemotherapy, stage of disease, and type of axillary lymph node surgery $(p<0.05)$. Of the surveys included in this final analysis of DBR diagnosed with breast cancer, this corresponds to $61.3 \%$ and $38.7 \%$ reporting no lymphedema and lymphedema symptoms, respectively. This is consistent with previous reports of the incidence of breast cancer associated lymphedema being anywhere from $5-50 \%$ (11-12). Data collected by the Lymph-ICF questionnaire demonstrated significant differences in total score $(\mathrm{p}<0.001)$ and in each of the five 


\begin{tabular}{|c|c|c|c|c|}
\hline & \multicolumn{3}{|c|}{ Lymphedema } & \multirow[b]{3}{*}{ p-value } \\
\hline & Yes (\%) & No (\%) & Total (\%) & \\
\hline & $\mathbf{n}=\mathbf{2 9 3}$ & $n=464$ & $\mathbf{n = 7 5 7}$ & \\
\hline Breast with cancer & & & & 0.54 \\
\hline Right & $124(42)$ & $212(46)$ & $336(45)$ & \\
\hline Left & $139(48)$ & $208(45)$ & $347(46)$ & \\
\hline Bilateral & $30(10)$ & $40(9)$ & $70(9)$ & \\
\hline Breast with surgery & & & & 0.89 \\
\hline Right & $108(37)$ & $177(38)$ & $285(38)$ & \\
\hline Left & $113(39)$ & $180(39)$ & $293(39)$ & \\
\hline Bilateral & $71(24)$ & $106(23)$ & $177(23)$ & \\
\hline Breast surgery & & & & 0.08 \\
\hline Lumpectomy & $119(41)$ & $225(48)$ & $344(45)$ & \\
\hline Mastectomy & $171(58)$ & $233(50)$ & $404(53)$ & \\
\hline Not applicable & $3(1)$ & $3(1)$ & $6(1)$ & \\
\hline Recurrence & $42(14)$ & $31(7)$ & $73(10)$ & $0.005^{*}$ \\
\hline No & $249(85)$ & $429(92)$ & $678(90)$ & \\
\hline Radiation & $208(71)$ & $317(68)$ & $525(69)$ & 0.61 \\
\hline No & $82(28)$ & $138(30)$ & $220(29)$ & \\
\hline Reconstruction & $63(22)$ & $111(24)$ & $174(23)$ & 0.47 \\
\hline No & $181(62)$ & $277(60)$ & $458961)$ & \\
\hline Bilateral & $46(16)$ & $61(13)$ & $107(14)$ & \\
\hline Axillary surgery & & & & $<0.001 *$ \\
\hline Sentinel node biopsy & $59(20)$ & $169(36)$ & $228(30)$ & \\
\hline ALND & $220(75)$ & $248(53)$ & $468(62)$ & \\
\hline Not applicable & $11(4)$ & $46(10)$ & $57(8)$ & \\
\hline Stage & & & & $<0.001 *$ \\
\hline 0 or DCIS & $4(1)$ & $15(3)$ & $19(2)$ & \\
\hline I & $60(20)$ & $187(40)$ & $247(33)$ & \\
\hline II & $101(34)$ & $122(26)$ & $223(30)$ & \\
\hline III & $86(29)$ & $76(16)$ & $162(22)$ & \\
\hline IV & $13(4)$ & $14(3)$ & $27(4)$ & \\
\hline Unknown & $28(10)$ & $38(8)$ & $66(9)$ & \\
\hline Hormone therapy & $204(70)$ & $303(65)$ & $507(67)$ & 0.22 \\
\hline No & $88(30)$ & $159(34)$ & $247(33)$ & \\
\hline Chemotherapy & $222(76)$ & $268(58)$ & $490(65)$ & $<0.001 *$ \\
\hline No & $71(24)$ & $195(42)$ & $266(35)$ & \\
\hline $\begin{array}{l}\text { Time since surgery (years) } \\
\text { Median }(95 \% \mathrm{CI})\end{array}$ & $10(6,14)$ & $9(5,14)$ & $9(5,14)$ & 0.17 \\
\hline
\end{tabular}

$D C I S=$ ductal carcinoma in situ, $A L N D=$ Axillary lymph node dissection, $C I=$ confidence interval *significant at $<0.05$ 
TABLE 2: Lymph-ICF Scores of Breast Cancer Survivors with and without lymphedema

\begin{tabular}{|c|c|c|c|c|c|c|}
\hline \multirow[b]{2}{*}{ Lymph-ICF Domains } & \multicolumn{2}{|c|}{$\begin{array}{c}\text { With } \\
\text { Lymphedema }\end{array}$} & \multicolumn{2}{|c|}{$\begin{array}{c}\text { Without } \\
\text { lymphedema }\end{array}$} & \multirow[b]{2}{*}{ z-value } & \multirow[b]{2}{*}{ p value } \\
\hline & $\mathbf{n}$ & $\begin{array}{c}\text { Median } \\
\text { (IQR) }\end{array}$ & n & $\begin{array}{c}\text { Median } \\
\text { (IQR) }\end{array}$ & & \\
\hline Physical function score & 239 & $12(5,36)$ & 390 & $4(1,11)$ & 10.39 & $<0.001 *$ \\
\hline 1. Heavy & 282 & $16(4,43)$ & 421 & $2(1,6)$ & 11.90 & $<0.001 *$ \\
\hline 2. Stiff & 278 & $9(2,33)$ & 423 & $3(1,7)$ & 7.75 & $<0.001^{*}$ \\
\hline 3. Swollen & 279 & $17(6,52)$ & 421 & $2(1,5)$ & 14.51 & $<0.001 *$ \\
\hline 4. Lost strength & 266 & $8(2,43)$ & 419 & $3(1,10)$ & 5.99 & $<0.001 *$ \\
\hline 5. Tingle & 273 & $7(1,30)$ & 423 & $3(1,9)$ & 5.53 & $<0.001 *$ \\
\hline 6. Hurt & 278 & $8(1,34)$ & 418 & $2(1,7)$ & 6.41 & $<0.001 *$ \\
\hline 7. Tensed skin & 272 & $4(1,26)$ & 406 & $2(0,5)$ & 5.08 & $<0.001 *$ \\
\hline Mental function score & 280 & $4(1,14)$ & 424 & $2(0,7)$ & 3.77 & $<0.001 *$ \\
\hline 8. Feel sad & 282 & $3(1,11)$ & 419 & $2(0,5)$ & 3.76 & $<0.001^{*}$ \\
\hline 9. Feel discouraged & 285 & $4(0,12)$ & 420 & $2(0,5)$ & 4.93 & $<0.001^{*}$ \\
\hline 10. Lack of self-confidence & 285 & $2(0,10)$ & 422 & $2(0,5)$ & 2.58 & 0.01* \\
\hline 11. Feel stressed & 286 & $3(1,16)$ & 420 & $2(0,8)$ & 2.59 & 0.01* \\
\hline Household activities score & 233 & $5(1,17)$ & 383 & $2(0,6)$ & 5.46 & $<0.001^{*}$ \\
\hline 12. Clean & 282 & $5(1,19)$ & 421 & $3(1,7)$ & 4.98 & $<0.001 *$ \\
\hline 13. Cook & 284 & $3(0,11)$ & 421 & $2(0,5)$ & 2.96 & $0.003 *$ \\
\hline 14. Iron & 258 & $3(1,11)$ & 406 & $2(0,5)$ & 3.44 & $0.001 *$ \\
\hline 15. Garden & 267 & $5(1,26)$ & 407 & $2(0,7)$ & 4.56 & $<0.001 *$ \\
\hline Mobility activities score & 220 & $9(2,23)$ & 363 & $2(0,9)$ & 7.97 & $<0.001^{*}$ \\
\hline 16. Tasks with arm elevated & 279 & $8(2,32)$ & 407 & $3(0,10)$ & 6.16 & $<0.001 *$ \\
\hline 17. Lift heavy objects & 286 & $12(2,42)$ & 412 & $2(0,12)$ & 7.62 & $<0.001 *$ \\
\hline 18. Sleep on affected side & 284 & $17(2,49)$ & 407 & $2(0,11)$ & 8.28 & $<0.001 *$ \\
\hline 19. Work on computer & 277 & $4(1,17)$ & 405 & $2(0,5)$ & 6.00 & $<0.001 *$ \\
\hline 20. Sunbathe & 230 & $3(0,28)$ & 353 & $1(0,6)$ & 4.61 & $<0.001 *$ \\
\hline 21. Drive a car & 281 & $2(0,6)$ & 403 & $1(0,3)$ & 2.92 & 0.003* \\
\hline 22. Walk & 284 & $2(0,5)$ & 406 & $1(0,3)$ & 2.30 & $0.02 *$ \\
\hline 23. Cycle & 247 & $2(0,7)$ & 382 & $1(0,4)$ & 2.34 & $0.02 *$ \\
\hline Life and social activities & 228 & $3(1,13)$ & 374 & $1(0,4)$ & 6.18 & $<0.001 *$ \\
\hline 24. Go on vacation & 283 & $2(0,5)$ & 414 & $1(0,4)$ & 1.29 & 0.20 \\
\hline 25. Perform Hobbies & 286 & $2(0,6)$ & 413 & $1(0,4)$ & 3.77 & $<0.001 *$ \\
\hline 26. Practice Sport & 286 & $2(0,9)$ & 415 & $1(0,4)$ & 3.77 & $<0.001 *$ \\
\hline 27. Wearing clothes of choice & 287 & $4(1,33)$ & 415 & $2(0,5)$ & 6.06 & $<0.001 *$ \\
\hline 28. Do a job & 263 & $2(0,8)$ & 382 & $1(0,4)$ & 4.46 & $<0.001 *$ \\
\hline 29. Do social activities & 285 & $2(0,6)$ & 417 & $1(0,4)$ & 2.33 & $0.02 *$ \\
\hline Lymph-ICF total score & 209 & $9(4,23)$ & 353 & $3(1,8)$ & 8.46 & $<0.001 *$ \\
\hline
\end{tabular}

$n=$ number of participants in the category, IQR=Interquartile range

*significant at $<0.05$ 
domains $(p<0.001)$ when comparing the lymphedema group to the non-lymphedema group. Each individual question scored statistically higher $(p<0.05)$ in the lymphedema group compared to the non-lymphedema group, except for "go on vacation" which was not statistically different between groups $(\mathrm{F}=1.29, \mathrm{p}=0.20)$. Table 2 provides a summary of the data including the number of partici-pants represented in each of the analyses.

\section{DISCUSSION}

This study on DBR in breast cancer survivors contributes to the growing evidence supporting an active lifestyle after a breast cancer diagnosis in all stages of disease. DBR has increased in popularity worldwide among breast cancer survivors, since 1998 when McKenzie demonstrated that participating in resistive exercise, such as DBR, did not induce or worsen lymphedema in breast cancer survivors (3). The IBCPC Participatory Dragon Boat Festival is the largest BCS competition worldwide allowing breast cancer survivors, with and without lymphedema, to participate in this competitive strenuous physical activity. International breast cancer survivors with lymphedema participating in DBR at this international competition had reduced function, limited activity, and restricted participation compared to participants without lymphedema. Although this multinational lymphedema group reported reduced QOL compared to participants without lymphedema, they still could participate in an aerobic and resistive intensive competitive exercise program.

This study included a large multicultural cohort investigating the impact of lymphedema on breast cancer survivors from DBR, which brought strength to this study. Community based breast cancer dragon boat racers were an integral part of the research team providing valuable input on the questionnaire, recruitment strategies, and data acquisition. Double measuring and entry of data, independent of each other, and not allowing the DBR research team to participate in the study reduced potential error and bias. Of particular note was that there was no significant difference in the groups on the question related to "how well you are able to go on vacations." This lack of difference between the groups is likely specific to the DBR competitors since participants were "on a vacation" and traveling for the event. Limita-tions to this study include missing data as a result of incomplete questionnaires, no objective measure to substantiate a lymphe-dema diagnosis, potential survey bias, and distributing surveys only written in the English language.

Previous DBR studies have not investigated lymphedema in such a large sample size from a multicultural setting. Prior studies using Lymph-ICF among breast cancer survivors with and without lymphedema had similar findings but were smaller, non-international studies $(13,14)$. Devoogt demonstrated significantly higher Lymph-ICF scores in a general group of women with breast cancer related lymphedema compared to women without lymphedema, which provided evidence on the construct validity of the Lymph-ICF in Belgium (13). Though our study was not a validity study, the results provide early evidence to support validity of the Lymph-ICF questionnaire. Using the Lymph-ICF scores to study international breast cancer survivors who participated in DBR demonstrated differences between breast cancer survivors with and without lymphedema. There is the question of the cultural attitudes and nationality affecting the patient's perception of lymphedema and their experience with this diagnosis. This study only touched on some of this variability. Yet, patient QOL is a concern that should be addressed by all health-care providers, regardless of location and cultural background. Though not directly comparable, it is notable the Lymph-ICF scores were lower in the DBR group compared to the general population of breast cancer survivors (13), indicating potential positive effect on QOL in women participating in DBR. Ray demonstrated the positive effect of DBR when QOL scores significantly improved from baseline in a group of breast cancer survivors participating in DBR (14). Regardless of the potential positive effects of participating in physical activity such as DBR, this study showed the negative impact 
lymphedema has on function, activity, and participation.

Medical providers need to increase their awareness of utilizing community-based programs, such as DBR, that promote participation in exercise and physical activity, which could further enhance a patient's recovery. DBR is a community activity that is available worldwide and can potentially improve QOL (5,10,14-20). Living a physically active lifestyle is essential for breast cancer survivors in all stages of disease, including advanced disease that is likely to reduce the risk of breast cancer recurrence and improve survival (21). Breast cancer survivors with and without lymphedema can participate in this activity without inducing or worsening lymphedema (3-5). Unfortunately, lymphedema negatively impacts QOL regardless of participation in physical activity such as DBR, although the impact could be less. Further research needs to be conducted to show that DBR, along with other types of exercise, may have a positive effect on QOL in breast cancer survivors in all stages of disease. Investigation should include the effect of duration, type of exercise, and intensity of participation. These variables were not included in this study. Interventions and strategies to prevent or reduce the risk of lymphedema require ongoing investigation. This study on DBR in breast cancer survivors contributes to the growing evidence supporting an active lifestyle in all stages of disease, including advanced disease, after a breast cancer diagnosis. A quarter of the participants in this study had Stage 3 or 4 disease.

International breast cancer survivors with lymphedema participating in DBR had reduced function, limited activity, and restricted participation compared to participants without lymphedema attending in the IBCPC Participatory Dragon Boat Festival. Although QOL was reduced in breast cancer survivors with lymphedema, the impact could be less in breast cancer survivors with lymphedema that participate in DBR. Breast cancer survivors can participate in an aerobic and resistive intensive competitive exercise program safely. Medical providers can better educate their patients accordingly on how to exercise safely and participate in an active lifestyle. There needs to be multiple exercise options for patients with breast cancer, including advanced disease, to enhance their mental status, physical ability and emotional QOL.

\section{ACKNOWLEDGMENTS}

The authors would like to give a very special thank you to the members of the local breast cancer survivor dragon boat race team from St. Paul, MN, the Dragon Divas, in addition to all the generous breast cancer survivors who participated in the study.

\section{FUNDINGSOURCE}

Research reported in this publication was supported in part by NIH P30 CA77598 utilizing the Masonic Cancer Center and University of Minnesota share resources and by the Eunice Kennedy Shriver National Institute of Child Health \& Human Development of the National Institutes of Health under Award Number K12HD055887. The content is solely the responsibility of the authors and does not necessarily represent the official views of the National Institutes of Health. The funders played no role in the design, conduct, or reporting of this study.

\section{CONFLICT OF INTEREST AND DISCLOSURE}

The authors declare no competing financial interests exist.

\section{REFERENCES}

\footnotetext{
1. International Society of Lymphology Execu-tive Committee: The diagnosis and treatment of peripheral lymphedema: 2020 Consensus Document of the international society of lymphology. Lymphology 53 (2020), 3-19.

2. Kent, H: Breast cancer survivors begin to challenge exercise taboos. CMAJ. 155 (1996),
} 
969-971.

3. McKenzie, DC: A breast in a boat - A race against breast cancer. CMAJ. 159 (1998), 376-378.

4. Harris, SR, SL Niesen-Vertommen: Challenging the myth of exercise-induced lymphedema following breast cancer: A series of case reports. J. Surg. Oncol. 74 (2000), 95-98. doi:10.1002/10969098(200006)74:2<95::AID-JSO3>3.0.CO;2-Q

5. Harris, SR: Were all in the same boat: A review of the benefits of dragon boat racing for women living with breast cancer. Evidence-based Complement Altern. Med. (2012), 167651. doi:

10.1155/2012/167651. Epub 2012 Jun 28.

6. Schmitz, KH: Balancing lymphedema risk:

Exercise versus deconditioning for breast cancer survivors. Exerc. Sport Sci. Rev. 38 (2010), 17-24. doi:10.1097/JES.0b013e3181c5cd5a

7. Schmitz, KH, RL Ahmed, AB Troxel, et al: Weight lifting for women at risk for breast cancer-related lymphedema a randomized trial. J. Am. Med. Assoc. 304 (2010), 2699-2705.

doi:10.1001/jama.2010.1837

8. Schmitz, KH, AB Troxel, A Cheville, et al: Physical activity and lymphedema (the PAL trial): Assessing the safety of progressive strength training in breast cancer survivors. Contemp. Clin. Trials. 30 (2009), 233-245. doi:10.1016/j.cct.2009.01.001

9. Kim, DS, YJ Sim, HJ Jeong, GC Kim: Effect of active resistive exercise on breast cancer-related lymphedema?: A randomized controlled trial. APMR. 91 (2010), 1844-1848. doi:10.1016/j.apmr.2010.09.008

10. Iacorossi, L, F Gambalunga, S Molinaro, et al: The effectiveness of the sport "Dragon Boat Racing" in reducing the risk of lymphedema incidence. Cancer Nurs. 42 (2019), 323-331.

11. Shah C, Vicini FA. Breast cancer-related arm lymphedema: Incidence rates, diagnostic techniques, optimal management and risk reduction strategies. Int J Radiat Oncol Biol Phys 2011;81:907-14.

12. Gillespie, Tessa $\mathrm{C}$ et al. "Breast cancer-related lymphedema: risk factors, precautionary measures, and treatments." Gland surgery vol. 7,4 (2018): 379 403. doi:10.21037/gs.2017.11.04 doi:10.1097/NCC.0000000000000615

13. Devoog,dt N, M Van Kampen, I Geraerts, et al: Lymphoedema Functioning, disability and health questionnaire (Lymph-ICF): Reliability and validity. Phys. Ther. 91 (2011), 944-957. doi:10.2522/ptj.20100087
14. Ray, HA, MJ Verhoef: Dragon boat racing and health-related quality of life of breast cancer survivors: A mixed methods evaluation. BMC Complement Altern. Med. 13 (2013), 1-12. doi:10.1186/1472-6882-13-205

15. Terry, M, E Nielsen: Living life to the limits: Dragon boaters and breast cancer. Can. Woman Stud. 21 (2002), 50-57.

16. Unruh, A, N Elvin: In the eye of the dragon: Women's experience of breast cancer and the occupation of dragon boat racing. Can. J. Occup. Ther. 71 (2004), 138-149.

17. Mitchell, T, C Yakiwchuk, K Griffin, R Gray, M Fitch: Survivor dragon boating: A vehicle to reclaim and enhance life after treatment for breast cancer. Health Care Women Int. 28 (2007), 122140. doi:10.1080/07399330601128445

18. Sabiston, CM, MH Mcdonough, PRE Crocker: Psychosocial experiences of breast cancer survivors involved in a dragon boat program: Exploring links to positive psychological growth. J. Sport Exec. Psychol. 29 (2007), 419-438.

19. Parry, DC: The contribution of dragon boat racing to women's health and breast cancer. Qual. Heal. Res. 18 (2008), 222-233.

20. McDonough, MH, CM Sabiston, S Ullrich-French: The development of social relationships, social support, and posttraumatic growth in a dragon boating team for breast cancer survivors. J. Sport Exerc. Psychol. 33 (2011), 627-648.

21. Hamer, J, E Warner: Lifestyle modifications for patients with breast cancer to improve prognosis and optimize overall health. CMAJ. 189 (2017), 268-274. doi:10.1503/cmaj.160464

Linda Koehler, PhD, PT, CLT-LANA

University of Minnesota

Divisions of Physical Therapy

and Rehabilitation Science

Department of Rehabilitation

Medicine University of Minnesota

Medical School Mayo Mail Code 388

420 Delaware St. SE

Minneapolis, MN 55455

Fax: koeh0139@umn.edu

Phone: 612-626-1502

Fax: 612-625-4274 